\title{
Knockdown of Cul4A increases chemosensitivity to gemcitabine through upregulation of TGFBI in lung cancer cells
}

\author{
MING-SZU HUNG ${ }^{1-3}$, I-CHUAN CHEN ${ }^{4,5}$, LIANG YOU ${ }^{6}$, DAVID M. JABLONS ${ }^{6}$, \\ YA-CHIN LI ${ }^{1}$, JIAN-HUA MAO ${ }^{7}$, ZHIDONG XU $^{6}$, MENG-JER HSIEH $^{8,9}$, YU-CHING LIN $^{1-3}$, \\ CHENG-TA YANG ${ }^{8,10}$, SHIH-TUNG LIU ${ }^{11}$ and YING-HUANG TSAI ${ }^{1,8}$
}

\begin{abstract}
${ }^{1}$ Division of Thoracic Oncology, Department of Pulmonary and Critical Care Medicine, Chang Gung Memorial Hospital, Chiayi Branch, Chiayi 61363; ${ }^{2}$ Department of Medicine, College of Medicine, Chang Gung University, Taoyuan 33302;

${ }^{3}$ Department of Respiratory Care, Chang Gung University of Science and Technology, Chiayi Campus;

${ }^{4}$ Department of Emergency Medicine, Chang Gung Memorial Hospital; ${ }^{5}$ Department of Nursing, Chang Gung University of Science and Technology, Chiayi Campus, Chiayi 61363, Taiwan, R.O.C.; ${ }^{6}$ Thoracic Oncology Laboratory,

Department of Surgery, Comprehensive Cancer Center, University of California, San Francisco, CA 94143;

${ }^{7}$ Life Sciences Division, Lawrence Berkeley National Laboratory, Berkeley, CA 94720, USA; ${ }^{8}$ Department of Respiratory Care, College of Medicine, Chang Gung University, Taoyuan 33302; ${ }^{9}$ Division of Pulmonary Infection and Critical Care,

Department of Pulmonary and Critical Care Medicine, Chang Gung Memorial Hospital, Chiayi 61363; ${ }^{10}$ Department of Pulmonary and Critical Care Medicine, Chang Gung Memorial Hospital, Taoyuan 33378; ${ }^{11}$ Department of Microbiology and Immunology, College of Medicine, Chang Gung University, Taoyuan 33302, Taiwan, R.O.C.
\end{abstract}

Received July 4, 2015; Accepted August 11, 2015

DOI: 10.3892/or.2015.4324

\begin{abstract}
Cullin 4A (Cul4A) promotes oncogenesis through overexpression and then ubiquitination-mediated proteolysis of tumor suppressors in various types of cancers. Transforming growth factor $\beta$-induced (TGFBI) has been implicated as a tumor suppressor, which enhances gemcitabine chemosensitivity in lung cancer cells. The present study aimed to investigate the association of TGFBI and Cul4A and the mechanism by which $\mathrm{Cu} 14 \mathrm{~A}$ regulates TGFBI. In addition, we also evaluated the therapeutic value of Cul4A RNAi using adenoviral transfection of Cul4A RNAi in nude mouse xenograft models. We observed that knockdown of Cul4A was associated with increased sensitivity to gemcitabine through upregulation of TGFBI in lung cancer cells. Cul4A regulated TGFBI through direct interaction and then ubiquitin-mediated protein degradation. In the nude mouse xenograft models, adenoviral transfection of Cul4A RNAi in combination with gemcitabine chemotherapy inhibited lung cancer tumor growth. As the result, combination of Cul4A RNAi with chemotherapy may provide a new approach to lung cancer treatment.
\end{abstract}

Correspondence to: Dr Ming-Szu Hung, Department of Pulmonary and Critical Care Medicine, Chang Gung Memorial Hospital, Chiayi Branch, 6 West Section, Jiapu Road, Puzi, Chiayi 61363, Taiwan, R.O.C.

E-mail:m12049@adm.cgmh.org.tw

Key words: lung cancer, Cul4A, TGFBI, gemcitabine

\section{Introduction}

Lung cancer is the leading cause of cancer-related deaths worldwide. The majority of non-small cell lung cancer (NSCLC) patients are in the advanced stage of the disease at the time of diagnosis. Systemic platinum-based chemotherapy is usually the treatment of choice for advanced lung cancer (1). Unfortunately, conventional chemotherapy regimens are hampered by limited efficacy, significant toxicity and a high relapse rate, indicating an urgent need for developing alternative therapeutic approaches such as targeted therapy. The development of tyrosine kinase inhibitors targeting mutant epidermal growth factor receptor (EGFR) is a good example of lung cancer targeted therapy (2). However, the identification of novel targets is still required in the future development of lung cancer therapy.

Cullin 4A (Cul4A) is a member of the evolutionarily conserved cullin protein family, which is related to the ubiquitin proteosome pathway. Overexpression of Cul4A has been observed in breast $(3,4)$, mesothelioma (5) and liver cancers (6). Cul4A has also been implicated in the ubiquitination and proteolysis of tumor suppressors, including p53 (7), NF2 (8), RASSF1A (9) and p21 (10,11), to promote oncogenesis. Our previous study demonstrated that the expression of Cul4A causes ubiquitination and destablization of p21 and p27. Consequently, downregulation of Cul4A expression resulted in G0/G1 arrest; and overexpression, increased growth of mesothelioma cells (5). Cul4A is also a critical gene for the survival of hematopoietic cells and development and growth of cancer cells (12). In a transgenic mouse study, conditional expression of $\mathrm{Cul4A}$ resulted in the formation of lung tumors $(11,13)$. 
Since Cul4A is overexpressed in cancers and is associated with oncogenesis, Cul4A can be a candidate for targeted therapy.

Transforming growth factor $\beta$-induced, $68-\mathrm{kDa}$ protein (TGFBI) is known as keratoepithelin or $\beta \mathrm{Ig}-\mathrm{h} 3$, and contains four conserved fasciclin-1 (FAS1) domains and a carboxyl-terminal Arg-Gly-Asp (RGD) integrin-binding sequence. TGFBI mediates integrin binding to extracellular matrix proteins such as collagen, laminin and fibronectin (14). Loss of TGFBI expression has been reported in several types of cancers including lung cancer (15), and it has been suggested to act as a tumor-suppressor gene (16). Additionally, overexpression of TGFBI in lung cancer cells increased the sensitivity to gemcitabine (17), and overexpression of TGFBI has also been reported to be associated with a better response to gemcitabine chemotherapy in lung cancer cells and patients. Although the Cul4A-DDB1 E3 ligase complex has been reported to ubiquitinate and degrade many tumor-suppressor proteins, it is unclear whether Cul4A causes ubiquitination of TGFBI to decrease its stability.

The present study aimed to investigate the association of TGFBI and $\mathrm{Cul} 4 \mathrm{~A}$ and the mechanism by which $\mathrm{Cul} 4 \mathrm{~A}$ regulates TGFBI. In addition, we also evaluated the therapeutic value of Cul4A RNAi using adenoviral transfection of Cul4A RNAi in a nude mouse xenograft model.

\section{Materials and methods}

Cell culture. The NSCLC cell lines A549 (ATCC CCL-185) and H460 (ATCC HTB-177) were purchased from the American Type Culture Collection (ATCC, Manassas, VA, USA). Both A549 and H460 cell lines were grown in complete RPMI-1640 growth medium supplemented with $10 \%$ fetal calf serum, $10 \mathrm{U} / \mathrm{ml}$ penicillin and $10 \mu \mathrm{g} / \mathrm{ml}$ streptomycin at $37^{\circ} \mathrm{C}$ and in $5 \% \mathrm{CO}_{2}$.

Retroviral production and transduction. The pBabe-puro retroviral vector (Addgene, Cambridge, MA, USA) was used to transduce the Cul4A gene, and the pSUPER-retro-puro vector (Oligoengine, Seattle, WA, USA) was used to express $\mathrm{Cul4A}$ shRNA. The resultant pBabe Cul4A vector expressing the Cul4A-myc-His fusion protein and the pSuper Cul4A vector expressing Cul4A shRNA were constructed as described previously (5). Retroviral vectors were transfected into the HEK 293 Phoenix ampho packaging cells (ATCC) using Fu-GENE6 transfection reagent (Roche, Lewes, UK). After transfection for $48 \mathrm{~h}$, the supernatant was filtered using a $0.45-\mathrm{mm}$ syringe filter. Retroviral infection was performed by adding the filtered supernatant to lung cancer cell lines cultured on $10-\mathrm{cm}$ dishes with $50 \%$ confluency in the presence of $8 \mu \mathrm{g} / \mathrm{ml}$ of Polybrene (Sigma-Aldrich, St. Louis, MO, USA). Six hours after infection, the culture medium was replaced with fresh medium. The infected cells were allowed to recover for $48 \mathrm{~h}$. Infected cells were selected by adding $1 \mathrm{mg} / \mathrm{ml}$ puromycin (Sigma-Aldrich) for $48 \mathrm{~h}$ and then maintained in complete medium with $0.5 \mathrm{mg} / \mathrm{ml}$ puromycin. Empty retroviral-infected stable cell lines were also produced following the above protocols (5).

Western blot analysis. Whole protein was extracted using Mammalian Protein Extraction Reagent (M-PER) from the cell lines added with Phosphatase Inhibitor Cocktail Set II (Calbiochem, San Diego, CA, USA) and Complete Protease Inhibitor Cocktails (Roche) according to the manufacturer's protocols. The proteins were separated on $4-15 \%$ gradient SDS-polyacrylamide gels and transferred to Immobilon-P membranes (Millipore, Billerica, MA, USA). The following primary antibodies were used: anti-Cul4A (Abcam, Cambridge, MA, USA), anti- $\beta$-actin (Sigma Chemical, St. Louis, MO, USA), anti-TGFBI (Santa Cruz Biotechnology, Inc., Santa Cruz, CA, USA) and anti-HA (Cell Signaling, Danvers, MA, USA). After being incubated with the appropriate secondary antibodies, the antigen-antibody complexes were detected using an ECL blotting analysis system (Amersham Pharmacia Biotech, Piscataway, NJ, USA). Densitometry of western blot analysis was determined by ImageJ (v1.44m for Windows; National Institutes of Health).

Semi-quantitative reverse transcription- $P C R(R T-P C R)$ analysis. RNA was extracted using the RNeasy Mini kit (Qiagen, Hilden, Germany) from cell pellets according to the manufacturer's instructions. Total RNA was then transcripted to cDNA using the iScript ${ }^{\mathrm{TM}}$ cDNA synthesis kit (Bio-Rad Laboratories, Munich, Germany). Reverse-transcribed cDNA (2 $\mu 1)$ was subjected to with a total volume of $20 \mu \mathrm{l}$ and the Bio-Rad CFX96 $^{\mathrm{TM}}$ quantitative PCR system (Bio-Rad Laboratories). The following primers were used for PCR: Cul4A, 5'-GCACT GGAGCGAGTACATCA-3' (sense) and 5'-CACATGCTTT GCGATCAGTT-3' (antisense); TGFBI, 5'-AGCCCTGCCAC CAAGAGAA-3' (sense) and 5'-CTCCGCTAACCAGGATTT CATC-3' (antisense); GAPDH, 5'-CATCCATGACAACTTTG GTATCGT-3' (sense) and 5'-CAGTCTTCTGGGTGGCAG TGA-3' (antisense). Amplification conditions were as follows: 1 cycle of $95^{\circ} \mathrm{C}$ for $5 \mathrm{~min}$ and 30 cycles of $95^{\circ} \mathrm{C}$ for $20 \mathrm{sec}, 58^{\circ} \mathrm{C}$ for $30 \mathrm{sec}, 72^{\circ} \mathrm{C}$ for $30 \mathrm{sec}$ and 1 cycle of $72^{\circ} \mathrm{C}$ for $5 \mathrm{~min}$ and then maintained at $4^{\circ} \mathrm{C}$.

Immunofluorescence. For immunofluorescence microscopy, the cells were grown on coverslips, fixed in cold methanol for $10 \mathrm{~min}$ at $-20^{\circ} \mathrm{C}$, and blocked with $2 \%$ bovine serum albumin for $30 \mathrm{~min}$. The cells were incubated with the primary anti-TGFBI (Santa Cruz Biotechnology, Inc.) antibody in $2 \%$ bovine serum albumin for $1 \mathrm{~h}$ at room temperature. The cells were washed with PBS and subsequently incubated with FITC-conjugated secondary antibodies in $2 \%$ bovine serum albumin for $1 \mathrm{~h}$ at room temperature. After washing with PBS, the cells were counterstained with DAPI and mounted in Vectashield (Vector Laboratories, Burlingame, CA, USA). Images were acquired using a TCS SP5 confocal microscope (Leica, Wetzlar, Germany). The intensity of TGFBI was quantified by MetaMorph ${ }^{\circledR}$ Microscopy Automation \& Image Analysis Software (Molecular Devices, Sunnyvale, CA, USA).

Cell viability assay. $\mathrm{H} 460$ and A549 stable cells $\left(1 \times 10^{4} / \mathrm{ml}\right)$ were cultured in 6-well plates for $48 \mathrm{~h}$ and then treated with the indicated concentration of gemcitabine for 72 or $96 \mathrm{~h}$ as indicated. Then, cells were trypsinized. The number of viable cells was counted by trypan blue dye exclusion using a hemocytometer. The result was expressed as a percentage, relative to 
the empty virus-transfected control groups. The half maximal inhibitory concentration $\left(\mathrm{IC}_{50}\right)$ value was determined using GraphPad Prism ${ }^{\circledR} \log$ (inhibitor) vs. response (variable slope) software (version 6; La Jolla, CA, USA).

Transfection of small interfering RNA (siRNA) and vectors. Pre-designed and validated TGFBI and universal negative control siRNA were purchased from Santa Cruz Biotechnology, Inc. Transfection was performed using Lipofectamine $^{\mathrm{TM}}$ RNAiMAX transfection reagent (Invitrogen Life Technologies, Carlsbad, CA, USA) according to the manufacturer's manual. The cells were plated in 6-well plates in antibiotic-free media, and transfection was performed with cells at $80 \%$ confluency with a final concentration of $50 \mathrm{nM}$ for each siRNA. At $96 \mathrm{~h}$ after transfection, the cells were treated with the indicated concentration of gemcitabine for $72 \mathrm{~h}$ and then the number of viable cells was counted.

The pCMV6-TGFBI-GFP (OriGene, Rockville, MD, USA) and empty pCDNA3 (Invitrogen Life Technologies) vectors were transfected with OmniFect ${ }^{\mathrm{TM}}$ transfection reagent (TransOMIC, Huntsville, AL, USA), according to the manufacturer's manual. The cells were plated in 6-well plates in antibiotic-free media, and transfection was performed with cells at $80 \%$ confluency with a final concentration of $0.5 \mu \mathrm{g}$ for each vector. At $96 \mathrm{~h}$ after transfection, the cells were treated with the indicated concentration of gemcitabine for $72 \mathrm{~h}$ and then the number of viable cells was counted.

Protein degradation assay. A protein degradation assay was used to evaluate the effects of Cul4A on the decay of TGFBI in lung cancer cells. Lung cancer cells that were retrovirally transfected with Cul4A shRNA and Cul4A were plated on $6-\mathrm{cm}$ culture dishes. At $80 \%$ confluency, the cells were exposed to $20 \mathrm{mg} / \mathrm{ml}$ of cycloheximide. Then, the cells were harvested at the indicated time points. Total cellular proteins were extracted and analyzed by western blot analysis using $\beta$-actin as a loading control.

Co-immunoprecipitation assay. 293T cells were transiently co-transfected with the pBabe-Cul4A-myc-his and pCMV6-TGFBI-GFP (OriGene) vectors with Lipofectamine 2000 transfection reagent (Invitrogen Life Technologies). Twenty-four hours after transfection, the cells were treated with $10 \mathrm{mM}$ of MG132 (Sigma-Aldrich) and then harvested in NP-40 lysis buffer [150 mM NaCl, $50 \mathrm{mM}$ Tris (pH 8.0), 1\% NP40], protease inhibitor and phosphatase inhibitor cocktail (Roche). Immunoprecipitation was performed by the Catch and Release v2.0 reversible immunoprecipitation system (Millipore) according to the manufacturer's protocols. Anti-GFP (OriGene) and anti-Myc tag (Cell Signaling, Danvers, MA, USA) antibodies were used for immunoprecipitation, respectively.

In vivo ubiquitination assay. 293T cells were cotransfected in combination with pBabe-Cul4A-myc-his and pCMV6-TGFBI-GFP (OriGene) with or without pRK5-HA-Ubiquitin-WT (Addgene). All cells were treated with $10 \mu \mathrm{M}$ of MG132 for $24 \mathrm{~h}$ prior to being lysed. Anti-GFP antibody was used for immunoprecipitation. Anti-HA antibody was used for western blot analysis.
Adenovirus expressing Cul4A RNAi. An adenovirus expressing Cul4A RNAi was generated with BLOCK-iT ${ }^{\mathrm{TM}}$ adenoviral RNAi expression system (Invitrogen, Life Technologies) according to the instructions from the manufacturer's manual. Briefly, double-stranded oligonucleotides that encode Cul4A siRNA were cloned into the $\mathrm{pENTR}^{\mathrm{TM}} / \mathrm{U} 6$ vector. The following Cul4A siRNAi sequences were used for cloning: 5'-GGUUUAUCCACGGUAAAGA-3' (5) and 5'-GCAGAA CUGAUCGCAAAGCAU-3' (18). The resulting clones were verified by DNA sequencing. This study used the $\mathrm{pAd} / \mathrm{BLOCK}-\mathrm{iT}^{\mathrm{TM}}$-DEST vector and a $\mathrm{pENTR}^{\mathrm{TM}} / \mathrm{U} 6$ entry clone in an LR recombination reaction to generate an expression clone containing the U6 RNAi cassette of Cul4A. After digesting the DNA with $P a c I$, the pAd/BLOCK-iT ${ }^{\mathrm{TM}}$-DEST expression construct was used to transfect the 293A cell line to produce an adenoviral stock. An empty virus was also generated using the same method. The adenoviral stock was amplified and titered using the Adeno- $\mathrm{X}^{\mathrm{TM}}$ Rapid Titer kit (Clontech Laboratories, Inc., Mountain View, CA, USA). Knockdown of Cul4A was confirmed by immunoblotting using the empty virus-transfected group as the control. The adenovirus was used for the xenograft study.

Nude mouse xenograft models. After approval from the Institutional Animal Care and Use Committee at Chang Gung Memorial Hospital, Chiayi, Taiwan, female Balb/c athymic nude mice of 5-6 weeks of age were housed under specific pathogen-free conditions. H460 or A549 lung cancer cells $\left(1 \times 10^{6}\right)$ in $100 \mu \mathrm{l}$ of serum-free RPMI-1640 medium and $20 \%$ Matrigel (BD Biosciences, San Jose, CA, USA) were injected subcutaneously into the flank areas of the mice. When tumors reached the mean size of $\sim 50-100 \mathrm{~mm}^{3}$, the mice were divided randomly into groups of five and injected with either AdCul4A or AdEV viruses with or without gemcitabine, at $120 \mathrm{mg} / \mathrm{kg}$ intraperitoneally (i.p.) weekly for three weeks. An intratumoral injection of $2 \times 10^{9}$ plaque forming units (PFU) of the AdCul4A virus or AdEV control virus was performed at days 0,7 and 14 from the day of injection. Tumors were measured with a caliper twice a week for 6 weeks, and the tumor volume was calculated by the formula $\left(\mathrm{Lx} \mathrm{W}^{2} / 2\right)$, where $\mathrm{L}$ represents the largest tumor diameter and $\mathrm{W}$ represents the smallest tumor diameter. Then, the mice were sacrificed, and tumors were excised for further studies at the indicated times.

Statistical analysis. The Student's t-test was used to compare variables in the different groups studied. Statistical analysis was carried out using SPSS (version 10.0; SPSS, Inc., Chicago, IL, USA). Significance was defined as $\mathrm{P}<0.05$ with two-sided analysis.

\section{Results}

Overexpression of Cul4A downregulates TGFBI expression in lung cancer cells. To study the correlation between Cul4A expression and TGFBI in lung cancer cells, we established stable cell lines expressing Cul4A using retroviral transfection with the pBabe-puro vector in H460 and A549 (H460 pBabeCul4A and A549 pBabeCul4A) lung cancer cells. In the stable cell lines overexpressing Cul4A, downregulation of TGFBI was observed when compared to the expression level 
A

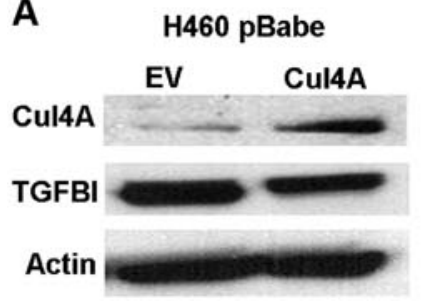

B

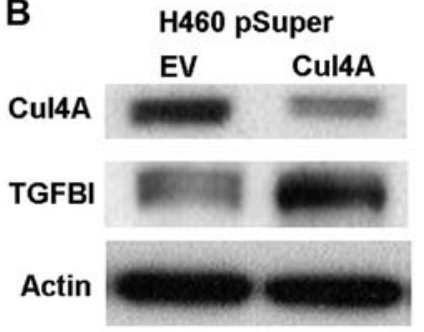

C

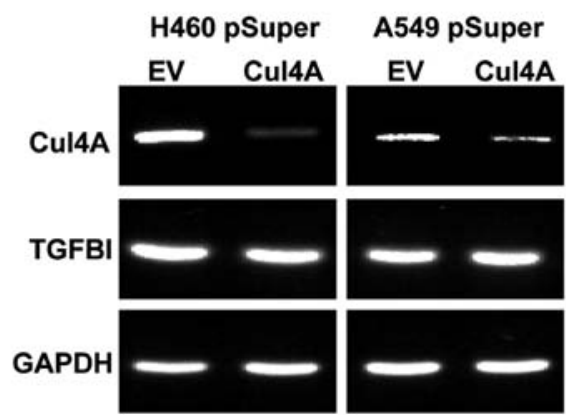

Figure 1. Western blot analysis for TGFBI in H460 and A549 lung cancer cells. (A) H460 and A549 lung cancer cells were stably transfected with pBabe Cul4A (Cul4A) for overexpression of Cul4A. The empty pBabe vector (EV)-transfected cells were used as the control group. (B) H460 and A549 lung cancer cells were stably transfected with pSuper Cul4A (Cul4A) for downregulation of Cul4A. The empty pSuper vector-(EV) transfected cells were used as the control group. $\beta$-actin was used as the internal control. (C) RT-PCR analysis for Cul4A and TGFBI in the H460 and A549 lung cancer cells stably transfected with pSuper Cul4A (Cul4A). The empty pSuper vector (EV)-transfected cells were used as the control group. GAPDH was used as the internal control.

in the empty vector-transfected stable cells (H460 pBabeEV and A549 pBabeEV) (Fig. 1A).

Downregulation of Cul4A upregulates TGFBI expression in lung cancer cells. To further study the association of Cul4A and TGFBI expression in lung cancer cells, knockdown of Cul4A expression in the H460 and A549 lung cancer cells was performed using retroviral transfection of Cul4A-specific shRNA with the pSuper-puro vector. In the Cul4A-knockdown H460 and A549 (H460 pSuperCul4A and A549 pSuperCul4A) lung cancer cells, upregulation of TGFBI was observed compared to the expression level in the empty vector-transfected stable cells (H460 pSuperEV and A549 pSuperEV) (Fig. 1B). To further evaluate whether upregulation of TGFBI is associated with increased transcription of TGFBI mRNA, RT-PCR was performed to evaluate the expression of Cul4A and TGFBI in the Cul4A-knockdown H460 and A549 lung cancer cells. The expression of TGFBI mRNA was not changed in both the Cul4A-knockdown lung cancer cell lines (Fig. 1C). Thus, Cul4A may not regulate TGFBI through transcriptional regulation of mRNA. Immunofluorescent
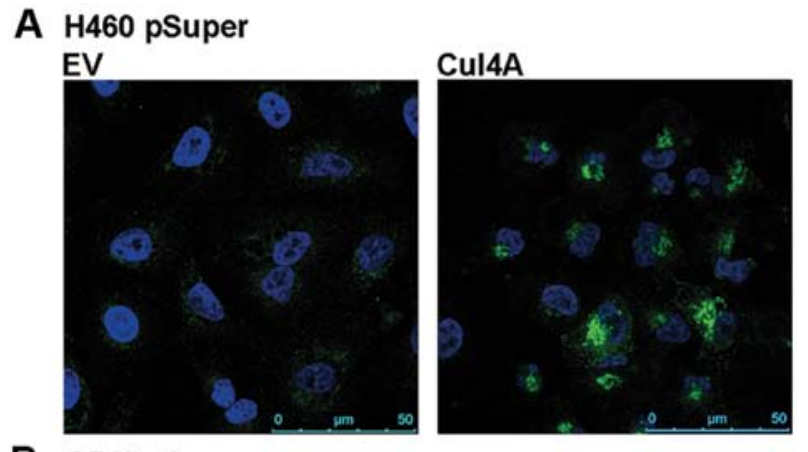

B A549 pSuper
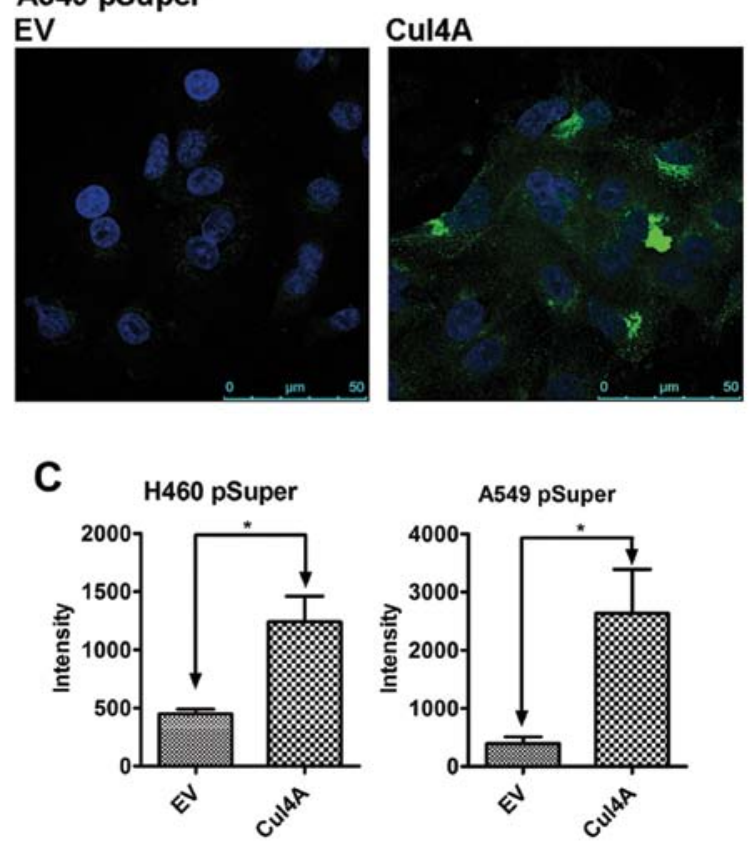

Figure 2. Immunofluorescent analysis of (A) H460 and (B) A549 lung cancer cells transfected with pSuper Cul4A (Cul4A) for downregulation of $\mathrm{Cu} 14 \mathrm{~A}$. The empty pSuper vector (EV)-transfected cells were used as the control group. TGFBI was stained green and nuclei were stained blue. Scale bar, $50 \mu \mathrm{m}$. Original magnification, $\times 200$. (C) The intensity of TGFBI in the each cells was quantified and shown as a mean \pm standard deviation in triple experiments. ${ }^{*} \mathrm{P}<0.05$.

analysis was also performed to evaluate the expression of TGFBI in the Cul4A-knockdown H460 (Fig. 2A) and A549 (Fig. 2B) (H460 pSuperCul4A and A549 pSuperCul4A) lung cancer cells. Upregulation of TGFBI was also observed in these cells when compared to the expression level in the empty vector-transfected stable cells (H460 pSuperEV and A549 pSuperEV), and TGFBI was mainly expressed in the cytoplasm and on the surface of the lung cancer cells studied.

Downregulation of Cul4A increases chemosensitivity to gemcitabine in lung cancer cells. We next studied the chemosensitivity to gemcitabine in the Cul4A-knockdown H460 and A549 lung cancer cells. We treated Cul4A-knockdown (H460 pSuperCul4A and A549 pSuperCul4A) and empty vector-transfected (H460 pSuperEV and A549 pSuperEV) lung cancer cells with the indicated concentrations of gemcitabine for $96 \mathrm{~h}$. Viable cells were counted and normalized to the cell number in the empty vector-transfected groups without treatment. $\mathrm{IC}_{50}$ values were determined in the $\mathrm{H} 460$ 

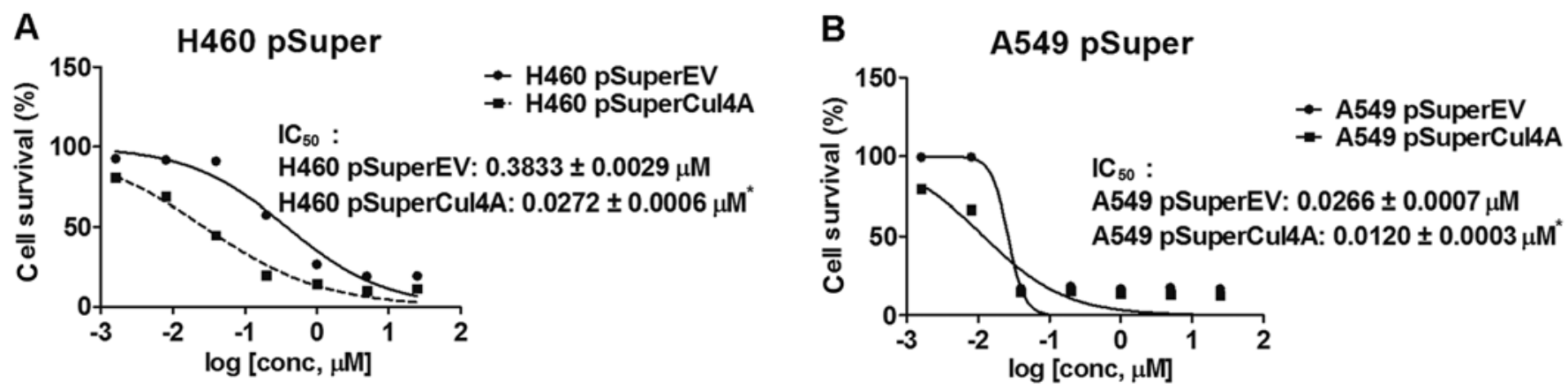

Figure 3. Sensitivity to gemcitabine of (A) H460 and (B) A549 lung cancer cells transfected with pSuper Cul4A (Cul4A) for downregulation of Cul4A. All groups of lung cancer cells were treated with the indicated concentrations of gemcitabine for $96 \mathrm{~h}$. The percentage of surviving cells was normalized to the empty vector-transfected groups without gemcitabine treatment and shown as mean \pm standard deviation in triple experiments. ${ }^{*} \mathrm{P}<0.05$.
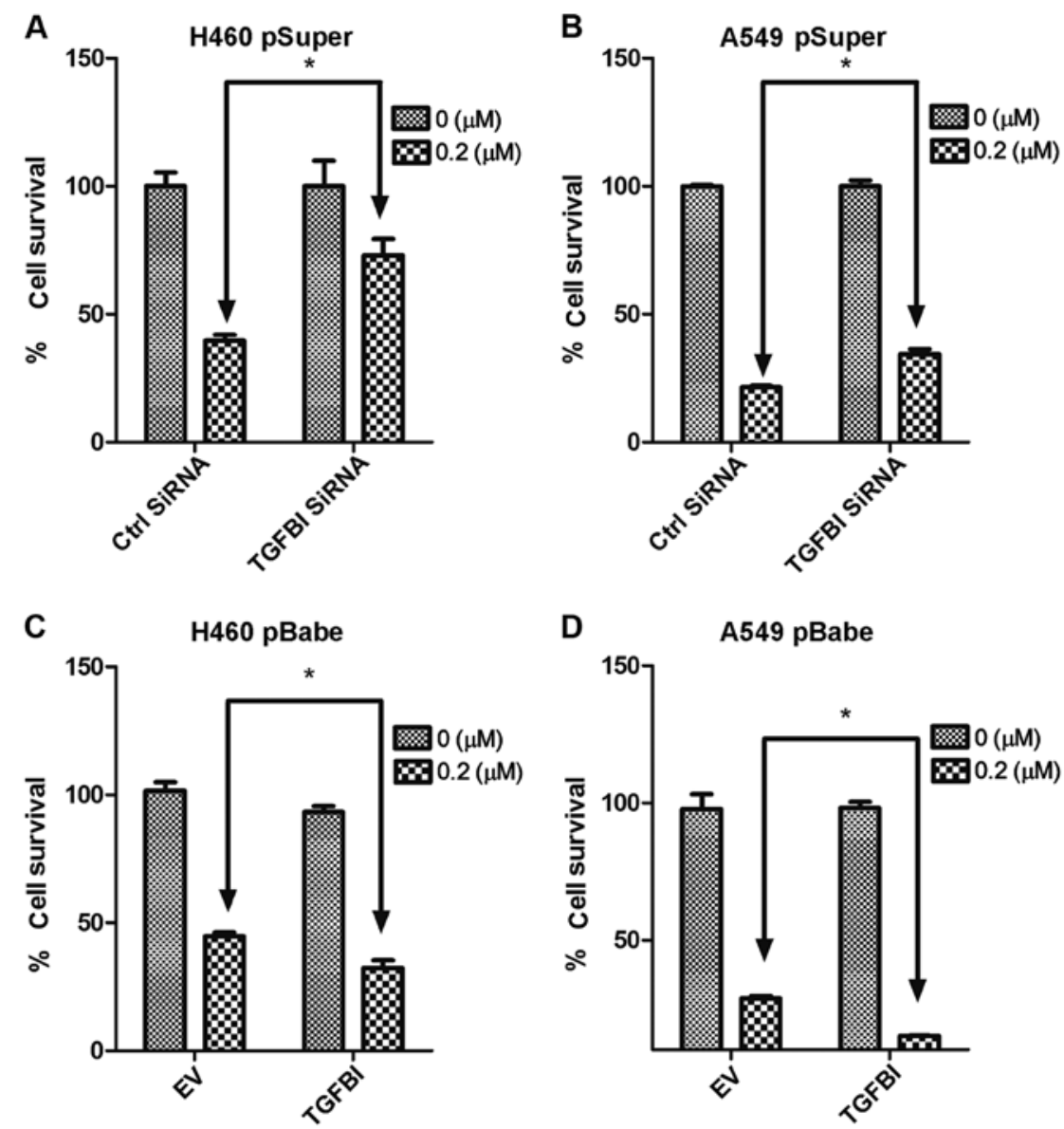

Figure 4. (A and B) Sensitivity to gemcitabine of the H460 and A549 lung cancer cells transfected with pSuper Cul4A for downregulation of Cul4A and TGFBI siRNA. Both H460 pSuper Cul4A and A549 pSuper Cul4A lung cancer cells were transfected with control (Ctrl siRNA) or TGFBI siRNA (TGFBI siRNA) for $96 \mathrm{~h}$ and then treated with the indicated concentrations of gemcitabine for $72 \mathrm{~h}$. (C and D) Sensitivity to gemcitabine of the H460 and A549 lung cancer cells transfected with pBabe Cul4A for overexpression of Cul4A and TGFBI. Both H460 pBabe Cul4A and A549 pBabe Cul4A lung cancer cells were transfected with empty (EV) or TGFBI expression (TGFBI) vectors for $96 \mathrm{~h}$ and then treated with the indicated concentrations of gemcitabine for $72 \mathrm{~h}$. The percentage of surviving cells was normalized to groups without gemcitabine treatment and shown as mean \pm standard deviation in triple experiments. "P<0.05.

pSuperCul4A $(0.0272 \pm 0.0006 \mu \mathrm{M})$, A549 pSuperCul4A $(0.0120 \pm 0.0003 \mu \mathrm{M}), \mathrm{H} 460 \mathrm{pSuperEV}(0.3833 \pm 0.0029 \mu \mathrm{M})$ and A549 pSuperEV $(0.0266 \pm 0.0007 \mu \mathrm{M})$ lung cancer cells. Significantly lower $\mathrm{IC}_{50}$ values were observed in the Cul4A-knockdown (H460 pSuperCul4A and A549 pSuperCul4A) cells when compared to the corresponding empty vector-transfected (H460 pSuperEV and A549 pSuperEV) lung cancer cells (Fig. 3A and B).
Downregulation of TGFBI decreases chemosensitivity to gemcitabine in Cul4A-knockdown lung cancer cells. To further evaluation the effects of TGFBI on chemosensitivity to gemcitabine, we downregulated TGFBI expression in the Cul4A-knockdown H460 pSuperCul4A and A549 pSuperCul4A lung cancer cells. All groups of cells were treated with $0.2 \mu \mathrm{M}$ of gemcitabine for $72 \mathrm{~h}$. Compared to the control siRNAtreated groups, significantly decreased chemosensitivity to 


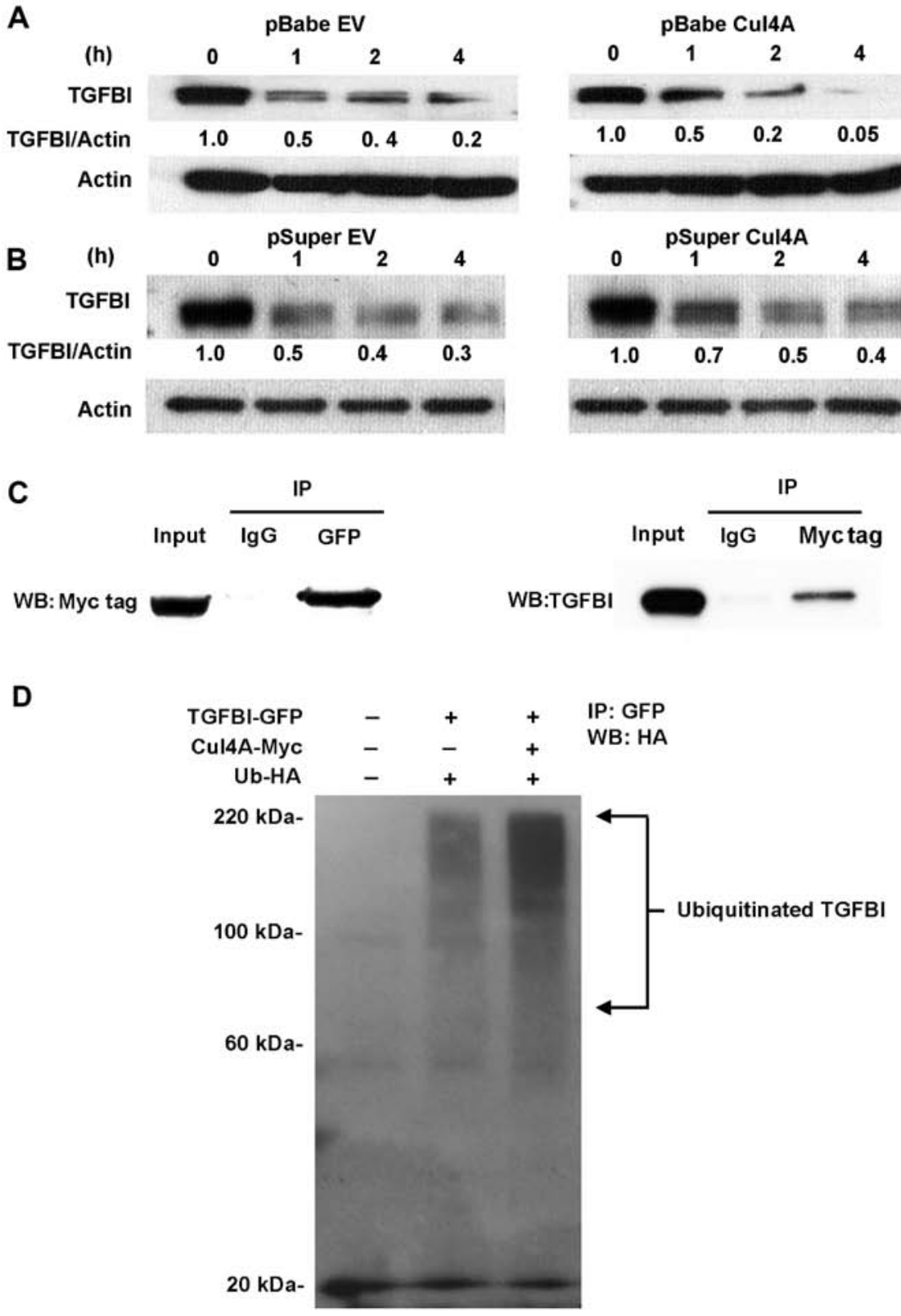

Figure 5. (A) Western blot analysis of TGFBI in the H460 lung cancer cell line. H460 lung cancer cells were stably transfected with the pBabe Cul4A overexpression vector or the empty pBabe EV vector. $\mathrm{H} 460$ cells were incubated with $20 \mu \mathrm{g} / \mathrm{ml}$ cycloheximide for the indicated time periods. (B) Western blot analysis for TGFBI in H460 lung cancer cell line. H460 lung cancer cells were stably transfected with the pSuper Cul4A to downregulate Cul4A or the empty pSuper EV vector. $\mathrm{H} 460$ cells were incubated with $20 \mu \mathrm{g} / \mathrm{ml}$ cycloheximide for the indicated time periods. (C) Reciprocal immunoprecipitation of Cul4A and TGFBI. The pBabe-Cul4A-myc-his and pCMV6-TGFBI-GFP vectors were cotransfected into 293T cells. Anti-GFP and anti-Myc tag antibodies were used for immunoprecipitation, respectively. (D) In vivo ubiquitination assay demonstrated that TGFBI is ubiquitinated by Cul4A in the cells.

gemcitabine was observed in the TGFBI siRNA-treated $\mathrm{H} 460$ pSuperCul4A (Fig. 4A) and A549 pSuperCul4A (Fig. 4B) lung cancer cells.

Overexpression of TGFBI increases chemosensitivity to gemcitabine in Cul4A-overexpressing lung cancer cells. The effects of TGFBI overexpression on chemosensitivity to gemcitabine were also studied. We overexpressed TGFBI in the $\mathrm{H} 460 \mathrm{pBabeCul} 4 \mathrm{~A}$ and A549 pBabeCul4A lung cancer cells. All groups of cells were treated with $0.2 \mu \mathrm{M}$ of gemcitabine for $72 \mathrm{~h}$. Compared to the empty vectortransfected groups, significantly increased chemosensitivity to gemcitabine was observed in the TGFBI-overexpressing H460
pBabeCul4A (Fig. 4C) and A549 pBabeCul4A (Fig. 4D) lung cancer cells.

Cul4A regulates TGFBI stability through protein degradation and ubiquitination. Since Cu14A-DDB1 E3 ligase complex has been reported to ubiquitinate and degrade many tumor-suppressor proteins, we thus studied whether Cul4A regulates TGFBI stability. A protein degradation assay was performed using the Cul4A-overexpressing $\mathrm{H} 460 \mathrm{pBabeCul} 4 \mathrm{~A}$ lung cancer cells. Compared to the empty vector-transfected H460 pBabeEV lung cancer cells, increased degradation of TGFBI was noted in the $\mathrm{H} 460 \mathrm{pBabeCul4A}$ lung cancer cells (Fig. 5A), which indicated that overexpression of Cul4A 
A

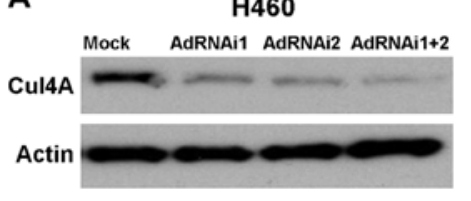

B
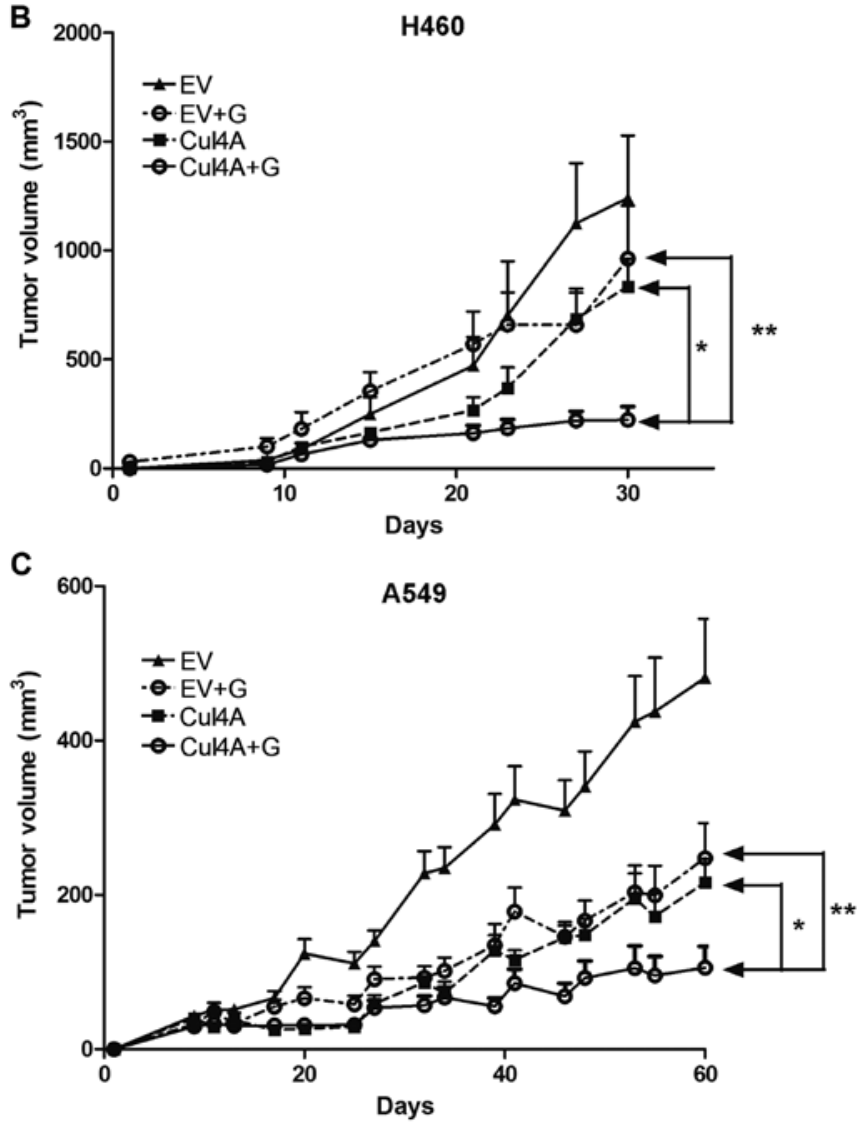

Figure 6. (A) Adenoviruses expressing Cul4A RNAi (AdRNAi1, AdRNAi2 or combination of both) were transfected into H460 and A549 lung cancer cells. Totally 200 MOI of each adenovirus was transfected. Mock, empty virus. Ninety-six hours post transfection, the cells were harvested and cell lysates were subjected to western blot analysis. (B) H460 lung cancer cell nude mouse xenograft model. Data points represent the average of tumor volume \pm standard deviation $\left(\mathrm{n}=6\right.$ in each group). ${ }^{*} \mathrm{P}=0.0014<0.05$ ${ }^{* *} \mathrm{P}=0.0345<0.05$. (C) A549 lung cancer cell nude mouse xenograft model. Data points represent the average of normalized tumor volume \pm standard deviation ( $\mathrm{n}=6$ in each group). ${ }^{*} \mathrm{P}=0.0241<0.05,{ }^{* *} \mathrm{P}=0.0241<0.05 ; \mathrm{EV}$, empty virus; Cul4A, Cul4A RNAi virus (100 MOI of each AdRNAil and AdRNAi2); G, gemcitabine.

is related to increased degradation of TGFBI. A protein degradation assay was also performed using Cul4A-knockdown H460 pSuperCul4A lung cancer cells. Compared to the empty vector-transfected H460 pSuperEV lung cancer cells, decreased degradation of TGFBI was noted in the H460 pSuperCul4A lung cancer cells (Fig. 5B), which indicated that downregulation of $\mathrm{Cul} 4 \mathrm{~A}$ is related to decreased degradation of TGFBI.

The interaction between Cul4A and TGFBI was also evaluated in our study. Reciprocal immunoprecipitation of Myc-tagged Cul4A and GFP-tagged TGFBI proteins was performed. Direct and preferential binding between the Cul4A and TGFBI proteins was noted (Fig. 5C). Since Cul4A exerts its functions through forming the multifunctional ubiquitin-protein ligase E3 complex by interacting with ROC1 and DDB1, we further performed in vivo ubiquitination assays to determine whether Cul4A is a TGFBI E3 ligase. $293 \mathrm{~T}$ cells were cotransfected with HA-tagged ubiquitin and Myc-tagged Cul4A in combination with GFP-tagged TGFBI. Our results showed that TGFBI was ubiquitinated by Cul4A (Fig. 5D).

Adenovirus-mediated transfection of Cul4A RNAi inhibits tumor growth in lung cancer xenograft models. Since the susceptibility of lung cancer cells to gemcitabine was elevated after the knockdown of Cul4A in the stable lung cancer cell lines (Fig. 3A and B), we further studied the therapeutic effects of Cul4A RNAi and the synergistic effects of Cul4A RNAi with gemcitabine in nude mouse xenograft models. Transient transfection of cells with Cul4A RNAi was performed in this model. Adenoviruses expressing Cul4A RNAi (AdRNAi1 and AdRNAi2) targeting different regions of the Cul4A gene were generated. Compared to the empty virus-transfected cells, the combination of both AdRNAi1 and AdRNAi2 viruses resulted in downregulation of Cul4A in the H460 and A549 lung cancer cells (Fig. 6A). Nude mouse xenografts derived from the H460 and A549 lung cancer cells were then established. Compared to the empty virus combined with gemcitabine and the Cul4A RNAi-transfected groups, significantly decreased tumor growth was observed in the groups which combined Cul4A RNAi and gemcitabine in the H460 (Fig. 6B) and A549 (Fig. 6C) lung cancer xenograft models.

\section{Discussion}

In the present study, we observed that knockdown of Cul4A enhanced chemosensitivity to gemcitabine in lung cancer cells. Upregulation of TGFBI was noted in the Cul4A-knockdown lung cancer cells and downregulation of TGFBI was noted in the Cul4A-overexpressing lung cancer cells. Further knockdown of TGFBI expression in the Cul4A-knockdown lung cancer cells decreased chemosensitivity. In contrast, overexpression of TGFBI in the Cul4A-overexpressing lung cancer cells increased chemosensitivity. Thus, the mechanisms by which Cul4A affects chemosensitivity to gemcitabine in lung cancer cells may be through regulation of TGFBI protein.

In addition, Cul4A knockdown significantly upregulated TGFBI protein levels and decreased its degradation, whereas overexpression of Cul4A markedly enhanced TGFBI protein ubiquitination and increased degradation of TGFBI protein. We also observed that Cul4A regulated TGFBI protein stability through direct interaction and ubiquitin mediated proteolysis. Thus, TGFBI is a target of the Cul4A E3 ligase complex. Cul4A has been reported to play a role in the ubiquitination and proteolysis of some well-defined tumor suppressors, including p53 (7), NF2 (8), RASSF1A (9), p27 (19), DDB2 (20) and p21 $(10,11)$. To the best of our knowledge, the association of Cul4A and TGFBI has not been previously reported.

Knockdown of Cul4A has been reported to increase chemosensitivity to cisplatin in lung cancer cells (13). Overexpression of Cul4A was also reported to participate in multiple drug resistance (MDR) in breast cancer cells through upregulation of MDR1/P-gp expression at both the transcription and protein 
levels, and knockdown of Cul4A increased chemosensitivity to P-gp substrate drugs, paclitaxel, vincristine and adriamycin in breast cancer cells (21). In the present study, we observed that knockdown of $\mathrm{Cul4A}$ is related to chemosensitivity to gemcitabine through upregulation of TGFBI in lung cancer cells for the first time. TGFBI-mediated chemosensitivity to gemcitabine has been reported to be related to proteolytic fragments derived from TGFBI-induced cell death through binding to the $\alpha v \beta 3$ integrin on the surface of NSCLC cells and the subsequent activation of caspase- 8 and caspase-3/7 signaling and resultant apoptosis of NSCLC cells (17). Gemcitabine is one of the most widely used drugs for the treatment of NSCLC. Cisplatin plus gemcitabine regimen has become a commonly used combination for advanced NSCLC (22) and maintenance therapy (23). When used as a first-line monotherapy, the objective response rate to gemcitabine is $16-22 \%$ (24). Thus, developing novel adjuvant chemotherapy in combination with gemcitabine chemotherapy is urgent. Our study may provide the rationale to improve the efficacy of gemcitabine chemotherapy as well as other chemotherapy drugs in NSCLC in combination with anti-Cul4A targeted therapy in the future.

Moreover, we also established an animal model to demonstrate the therapeutic value of Cul4A RNAi. We observed that tumor size was significantly decreased in the group of mice treated with Cul4A RNAi and gemcitabine. To date, there is no Cul4A-specific small compound reported. RNAi is a potential new class of pharmaceutical drugs. RNAibased therapeutics can offer a powerful method for rapidly identifying specific and potent inhibitors of disease targets from all molecular classes. The broad potential application of RNAi therapeutics has been demonstrated by numerous proof-of-concept studies in animal models of human diseases (25). The use of adenoviral vectors is considered to be a very powerful tool in cancer gene therapy as they have been shown to transduce genes efficiently into many types of cancer cells (26). In the present study, we designed recombinant adenoviral vectors encoding siRNAs against Cul4A and investigated their efficacy in regard to the suppression of Cul4A expression in cancer cells and consequent antitumor potential. Through in vivo studies, our results support the feasibility of adenoviral-mediated transfer of Cul4A RNAi for treatment of NSCLC and other cancers in the future.

In summary, the present study showed that knockdown of Cul4A is associated with increased sensitivity to gemcitabine through upregulation of TGFBI in NSCLC cells. Cul4A regulates TGFBI through direct interaction and then ubiquitin-mediated protein degradation. In nude mouse xenograft models, adenoviral-mediated transfer of Cul4A RNAi in combination with gemcitabine chemotherapy inhibited NSCLC tumor growth. Therefore, combination of Cul4A RNAi with chemotherapy may provide a new approach to lung cancer treatment.

\section{Acknowledgements}

The present study was supported by grants from the Chang Gung Memorial Hospital (CMRPG6E0081, NMRPD1B1331 and NMRPD1B1331) and the National Science Council, Taiwan (101-2314-B-182-086-MY2). We would like to acknowledge the Leica SP5II confocal microscope service provided by the
Expensive Advanced Instrument Core Laboratory, Department of Medical Research and Development, Chang Gung Memorial Hospital at Chiayi.

\section{References}

1. Azzoli CG, Baker S Jr, Temin S, Pao W, Aliff T, Brahmer J, Johnson DH, Laskin JL, Masters G, Milton D, et al; American Society of Clinical Oncology: American Society of Clinical Oncology Clinical Practice Guideline update on chemotherapy for stage IV non-small-cell lung cancer. J Clin Oncol 27: 6251-6266, 2009.

2. Maemondo M, Inoue A, Kobayashi K, Sugawara S, Oizumi S, Isobe $\mathrm{H}$, Gemma A, Harada M, Yoshizawa H, Kinoshita I, et al; North-East Japan Study Group: Gefitinib or chemotherapy for non-small-cell lung cancer with mutated EGFR. N Engl J Med 362: 2380-2388, 2010.

3. Abba MC, Fabris VT, Hu Y, Kittrell FS, Cai WW, Donehower LA, Sahin A, Medina D and Aldaz CM: Identification of novel amplification gene targets in mouse and human breast cancer at a syntenic cluster mapping to mouse ch8A1 and human ch13q34. Cancer Res 67: 4104-4112, 2007.

4. Melchor L, Saucedo-Cuevas LP, Muñoz-Repeto I, RodríguezPinilla SM, Honrado E, Campoverde A,Palacios J, Nathanson KL, García MJ and Benítez J: Comprehensive characterization of the DNA amplification at 13q34 in human breast cancer reveals TFDP1 and CUL4A as likely candidate target genes. Breast Cancer Res 11: R86, 2009.

5. Hung MS, Mao JH, Xu Z, Yang CT, Yu JS, Harvard C, Lin YC, Bravo DT, Jablons DM and You L: Cul4A is an oncogene in malignant pleural mesothelioma. J Cell Mol Med 15: 350-358, 2011.

6. Yasui K, Arii S, Zhao C, Imoto I, Ueda M, Nagai H, Emi M and Inazawa J: TFDP1, CUL4A, and CDC16 identified as targets for amplification at $13 \mathrm{q} 34$ in hepatocellular carcinomas. Hepatology 35: 1476-1484, 2002.

7. Nag A, Bagchi S and Raychaudhuri P: Cul4A physically associates with MDM2 and participates in the proteolysis of $\mathrm{p} 53$. Cancer Res 64: 8152-8155, 2004.

8. Huang J and Chen J: VprBP targets Merlin to the Roc1-Cul4A-DDB1 E3 ligase complex for degradation. Oncogene 27: 4056-4064, 2008.

9. Jiang X, Gu X, Liu Y, Ding F, Gu X, Huan Y, Ren L and Wang Y: Molecular cloning and expression analysis of evolutionarily conserved stathmin from Gekko japonicus spinal cord. Indian J Biochem Biophys 46: 289-293, 2009.

10. Nishitani H, Shiomi Y, Iida H, Michishita M, Takami T and Tsurimoto T: CDK inhibitor p21 is degraded by a PCNA coupled Cul4 DDB1Cdt2 pathway during S phase and after UV irradiation. J Biol Chem 43: 29045-29052, 2008.

11. Li T, Hung MS, Wang Y, Mao JH, Tan JL, Jahan K, Roos H, Xu Z, Jablons DM and You L: Transgenic mice for cre-inducible overexpression of the Cul4A gene. Genesis 49: 134-141, 2011

12. Miranda-Carboni GA, Krum SA, Yee K, Nava M, Deng QE, Pervin S, Collado-Hidalgo A, Galic Z, Zack JA, Nakayama K, et al: A functional link between Wnt signaling and SKP2-independent p27 turnover in mammary tumors. Genes Dev 22: 3121-3134, 2008.

13. Yang YL, Hung MS, Wang Y, Ni J, Mao JH, Hsieh D, Au A, Kumar A, Quigley D, Fang LT, et al: Lung tumourigenesis in a conditional Cul4A transgenic mouse model. J Pathol 233: 113-123, 2014.

14. Kim JE, Kim SJ, Lee BH, Park RW, Kim KS and Kim IS: Identification of motifs for cell adhesion within the repeated domains of transforming growth factor-beta-induced gene, betaig-h3. J Biol Chem 275: 30907-30915, 2000.

15. Zhao Y, El-Gabry M and Hei TK: Loss of Betaig-h3 protein is frequent in primary lung carcinoma and related to tumorigenic phenotype in lung cancer cells. Mol Carcinog 45: 84-92, 2006.

16. Zhao YL, Piao CQ and Hei TK: Downregulation of Betaig-h3 gene is causally linked to tumorigenic phenotype in asbestos treated immortalized human bronchial epithelial cells. Oncogene 21: 7471-7477, 2002.

17. Irigoyen M, Pajares MJ, Agorreta J, Ponz-Sarvisé M, Salvo E, Lozano MD, Pío R, Gil-Bazo I and Rouzaut A: TGFBI expression is associated with a better response to chemotherapy in NSCLC. Mol Cancer 9: 130, 2010. 
18. Jiang L, Rong R, Sheikh MS and Huang Y: Cullin-4A DNA damage-binding protein $1 \mathrm{E} 3$ ligase complex targets tumor suppressor RASSF1A for degradation during mitosis. J Biol Chem 286: 6971-6978, 2011.

19. Bondar T, Kalinina A, Khair L, Kopanja D, Nag A, Bagchi S and Raychaudhuri P: Cul4A and DDB1 associate with Skp2 to target p27Kip1 for proteolysis involving the COP9 signalosome. Mol Cell Biol 26: 2531-2539, 2006.

20. El-Mahdy MA, Zhu Q, Wang QE, Wani G, Praetorius-Ibba M and Wani AA: Cullin 4A-mediated proteolysis of DDB2 protein at DNA damage sites regulates in vivo lesion recognition by XPC. J Biol Chem 281: 13404-13411, 2006.

21. Wang Y, Ma G, Wang Q, Wen M, Xu Y, He X, Zhang P, Wang Y, Yang T, Zhan $\mathrm{P}$, et al: Involvement of CUL4A in regulation of multidrug resistance to $\mathrm{P}$-gp substrate drugs in breast cancer cells. Molecules 19: 159-176, 2013.

22. Inal A, Kos FT, Algin E, Yildiz R, Dikiltas M, Unek IT, Colak D, Elkiran ET, Helvaci K, Geredeli C, et al: Gemcitabine alone versus combination of gemcitabine and cisplatin for the treatment of patients with locally advanced and/or metastatic pancreatic carcinoma: A retrospective analysis of multicenter study. Neoplasma 59: 297-301, 2012.
23. Brodowicz T, Krzakowski M, Zwitter M, Tzekova V, Ramlau R, Ghilezan N, Ciuleanu T, Cucevic B, Gyurkovits K, Ulsperger E, et al; Central European Cooperative Oncology Group CECOG: Cisplatin and gemcitabine first-line chemotherapy followed by maintenance gemcitabine or best supportive care in advanced non-small cell lung cancer: A phase III trial. Lung Cancer 52: 155-163, 2006.

24. Ricci S, Antonuzzo A, Galli L, Tibaldi C, Bertuccelli M, Lopes Pegna A, Petruzzelli S, Algeri R, Bonifazi V, Fioretto ML, et al: Gemcitabine monotherapy in elderly patients with advanced non-small cell lung cancer: A multicenter phase II study. Lung Cancer 27: 75-80, 2000.

25. Bumcrot D, Manoharan M, Koteliansky V and Sah DW: RNAi therapeutics: A potential new class of pharmaceutical drugs. Nat Chem Biol 2: 711-719, 2006.

26. Uchida H, Tanaka T, Sasaki K, Kato K, Dehari H, Ito Y, Kobune M, Miyagishi M, Taira K, Tahara $\mathrm{H}$, et al: Adenovirus-mediated transfer of siRNA against survivin induced apoptosis and attenuated tumor cell growth in vitro and in vivo. Mol Ther 10: 162-171, 2004. 